\title{
Inhibition of microglial activity alters spinal wide dynamic range neuron discharge and reduces microglial Toll-like receptor 4 expression in neuropathic rats
}

\author{
Samad Nazemi, ${ }^{* \dagger}$ Homa Manaheji, ${ }^{\dagger *}$ Syyed Mohammad Noorbakhsh, ${ }^{\S}$ Jalal Zaringhalam, ${ }^{\dagger *}$ \\ Mehdi Sadeghi, ${ }^{\mathbb{T}}$ Mohammad Mohammad-Zadeh* and Abbas Haghparast \\ *Department of Physiology, Sabzevar University of Medical Sciences, Sabzevar, Iran, ${ }^{\dagger}$ Department of Neurophysiology, \\ Shahid Beheshti University of Medical Sciences, Tehran, Iran, ${ }^{\ddagger}$ Neuroscience Research Center, Shahid Beheshti \\ University of Medical Sciences, Tehran, Iran, ${ }^{\S}$ Science Beam Institute, Tehran, Iran and ${ }^{\Uparrow}$ Department of Physiology, \\ Bushehr University of Medical Sciences, Bushehr, Iran
}

\begin{abstract}
SUMMARY
It is believed that neuropathic pain results from aberrant neuronal discharges although some evidence suggests that the activation of glia cells contributes to pain after an injury to the nervous system. This study aimed to evaluate the role of microglial activation on the hyper-responsiveness of wide dynamic range neurons (WDR) and Toll-like receptor 4 (TLR4) expressions in a chronic constriction injury (CCI) model of neuropathic pain in rats. Adult male Wistar rats $(230 \pm 30 \mathrm{~g})$ underwent surgery for induction of CCI neuropathy. Six days after surgery, administration of minocycline $(10,20$, and $40 \mathrm{mg} / \mathrm{kg}$, i.p.) was initiated and continued until day 14. After administration of the last dose of minocycline or saline, a behavioral test was conducted, then animals were sacrificed and lumbar segments of the spinal cord were collected for Western blot analysis of TLR4 expression. The electrophysiological properties of WDR neurons were investigated by single unit recordings in separate groups. The findings showed that after CCI, in parallel with thermal hyperalgesia, the expression of TLR4 in the spinal cord and the evoked response of the WDR neurons to electrical, mechanical, and thermal stimulation significantly increased. Post-injury administration of minocycline effectively decreased thermal hyperalgesia, TLR4 expression, and hyperresponsiveness of WDR neurons in CCI rats. The results of this study indicate that post-injury, repeated administration of minocycline attenuated neuropathic pain by suppressing microglia activation and reducing WDR neuron hyperresponsiveness. This study confirms that post-injury modulation of microglial activity is a new strategy for treating neuropathic pain.
\end{abstract}

Correspondence: H Manaheji, Department of Neurophysiology, Shahid Beheshti University of Medical Sciences, PO-Box 19615-1178, Tehran, Iran. Email: Hshardimanaheji@yahoo.com

Received 15 December 2014; revision 15 April 2015; accepted 18 April 2015.

(C) 2015 Wiley Publishing Asia Pty Ltd
Key words: hyperalgesia, microglia, neuropathic pain. toll-like receptor 4, WDR neuron.

\section{INTRODUCTION}

Neuropathic pain is defined as pain caused by a lesion or dysfunction in the peripheral or central nervous system, which is characterized by hyperalgesia (increased responsiveness to painful stimuli), allodynia (pain perceived in response to normally innocuous stimuli), and spontaneous pain. ${ }^{1,2}$ It was previously believed that pain arises primarily from neuronal dysfunction. However, new studies show that the activation of neuroimmune responses may also contribute to pain after injury to the nervous system. Glial cells, including both astroglia and microglia, are resident within the central nervous system and are involved in mediating the inflammatory processes after nerve injury. In particular, microglia are directly responsible for the initiation of neuropathic pain. $^{3-5}$

Preemptive or post-injury delivery of glial inhibitors results in different effects on neuropathic pain phenomenology. ${ }^{6-9}$ Previous studies have shown that preemptive treatment with glial inhibitors, such as minocycline and pentoxifylline, can effectively attenuate neuropathic pain symptoms and decrease the expression of different markers of glial activation including ionized calciumbinding adapter molecule 1 (Iba1), glial fibrillary acidic protein (GFAP), and Toll-like receptor 4 (TLR4). ${ }^{6,8,10,11}$ Toll-like receptor 4 is expressed predominantly in microglia and plays an important role in microglial activation. ${ }^{12}$ TLR4 antagonists evoke relief from both thermal hyperalgesia and mechanical allodynia in mice with painful neuropathy. ${ }^{13}$ Moreover, the expression of TLR4 on spinal cord microglia increases significantly in response to nerve injury. ${ }^{14-16}$

There are a limited number of electrophysiological studies that have investigated the effect of peripheral nerve injury on the response of wide dynamic range (WDR) neurons. ${ }^{17-21}$ Electrophysiological studies in different animal models of neuropathy have demonstrated abnormal hyper-responsiveness or receptive field enlargement, ${ }^{22,23}$ and increased spontaneous activity of WDR neurons. ${ }^{24}$ All of these abnormal features indicate a pro- 
nounced hyperexcitability in WDR neurons which explains the neuronal basis for the behavioral signs of allodynia and hyperalgesia in neuropathy. ${ }^{25}$

Previous electrophysiological studies have focused on the altered hyperexcitability of WDR neurons, and few attempts have been made to clarify the effect of glial activation and inhibition on this abnormal hyperexcitability. In addition, the effect of postinjury administration of minocycline on activated glia cells is not fully understood. Therefore, the aim of this study was to determine the effects of microglial activation and inhibition on TLR4 receptor expression and the electrophysiological properties of WDR neurons in the dorsal horn of the spinal cord in chronic constriction nerve injury in rats.

\section{RESULTS}

Chronic post-injury administration of minocycline attenuates thermal hyperalgesia in CCI-induced neuropathic rats

The successful induction of neuropathic pain symptoms was approved by the Hargreaves test done on postoperative day (POD) 6. The effects of post-injury administration of minocycline from POD 6-14 on thermal hyperalgesia were evaluated on POD14. One-way ANOVA indicated significant changes in paw withdrawal latencies $(\mathrm{PWL})\left(F_{4,35}=53.65, P<0.001\right)$ (Fig. 1). Tukey's post-hoc test also revealed that administration of minocycline (40 mg/kg; intraperitoneal (i.p.)) from POD 6-14 significantly attenuated thermal hyperalgesia in the ipsilateral hind paw $(P=0.001)$.

In this study, a $60 \%$ decrease in paw withdrawal latency (PWL) response to baseline was considered as the threshold for induction of successful neuropathic pain on day 6 after CCI $(85 \%)$. Therefore, animals that showed less than a $60 \%$ decrease in PWL (15\%) were omitted from the study (data not shown).

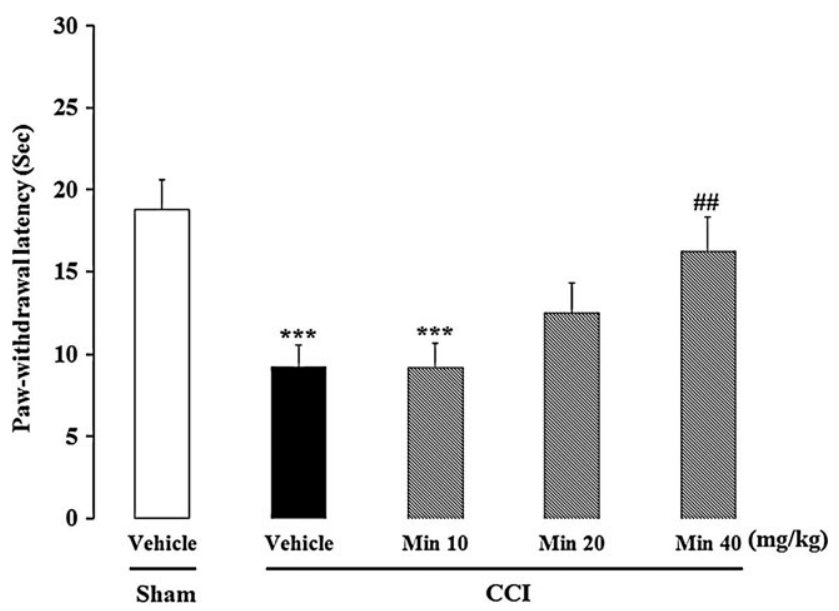

Fig. 1 The effect of post-injury repeated administration of minocycline $(10,20$, and $40 \mathrm{mg} / \mathrm{kg}$; i.p.) from POD 6 to POD 14 on thermal hyperalgesia. The data are presented as mean $\pm \operatorname{SEM}(n=8)$. Inter-group differences were analyzed by ANOva followed by Bonferroni's multiple comparison tests. Min, minocycline. ${ }^{* * *} P<0.001$, significant difference with sham + vehicle group; \#\#P $<0.01$, significant difference with CCI + Vehicle group.
Chronic post-injury administration of minocycline decreased TLR4 expression

The effects of post-injury administration of minocycline (10, 20, and $40 \mathrm{mg} / \mathrm{kg}$; i.p. from POD 6-14) on TLR4 expression were evaluated on POD14. One-way ANOvA indicated significant changes in TLR4 expression $\left(F_{4,35}=47.65, P<0.001\right)$ (Fig. 2$)$. Also, Tukey's post-hoc test revealed that expression of TLR4 increased in the CCI group in comparison to the sham-operated group $(P<0.0001)$. In addition, in groups that received minocycline 20 and $40 \mathrm{mg} / \mathrm{kg}$, the expression of TLR4 significantly decreased compared to the CCI group $(P<0.001)$.

Since the dose of $40 \mathrm{mg}$ minocycline showed significant effects on both the behavioral and molecular study, only this dose was used in the electrophysiological study.

\section{Changes in electrophysiological characteristics of WDR neurons following CCI}

A total of 25 WDR neurons (CCI group, 11 neurons from seven rats; sham-operated group, 14 neurons from 11 rats) were employed. The receptive fields of all neurons were defined in the toe/plantar region of the ipsilateral hind paw and displayed characteristics of WDR neurons that responded to both noxious and innocuous stimuli. All WDR neurons employed in the study were located at approximately similar depths from the surface of the spinal cord (300-1000 $\mu \mathrm{m})$. Likewise, the neuronal thresholds for $\mathrm{C}$ - and $\mathrm{A} \delta$-fibre activation were similar in all animal groups (data not shown).

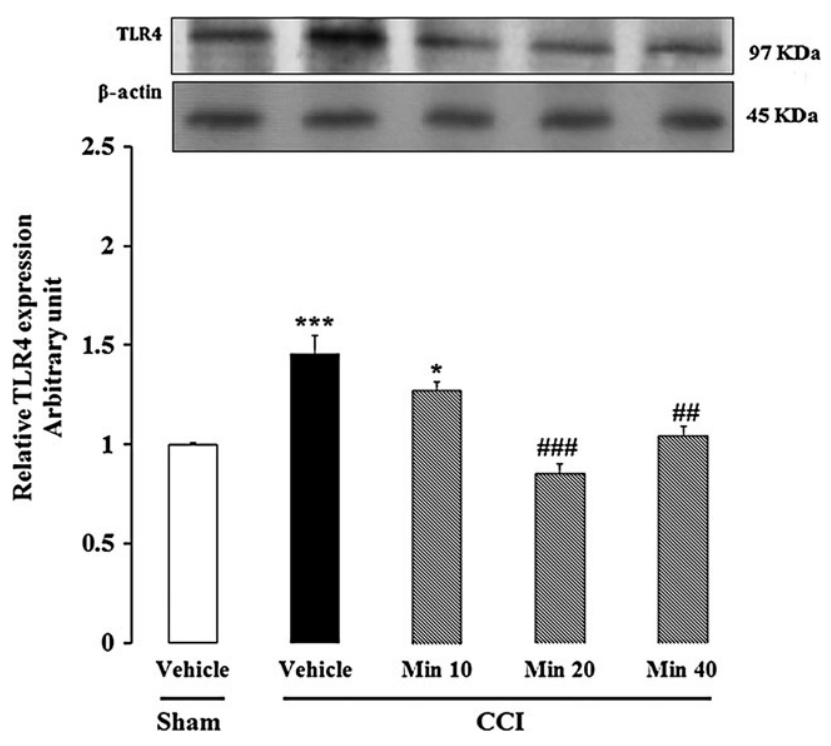

Fig. 2 The expression of TLR4 on the dorsal horn of spinal cord was assessed by immunoblot analysis. Equal amounts $(60 \mu \mathrm{g} / \mathrm{lane})$ of each sample were loaded on SDS-PAGE gel. The results were then normalized to beta actin and express as mean $\pm \operatorname{SEM}(n=6)$. After CCI, the expression of TLR4 was increased. Additionally, minocycline $(20,40 \mathrm{mg} / \mathrm{kg}$; i.p.) significantly decreased the expression of the TLR4 receptor in CCI animals. Representative immunoblots are shown above the graph. The experiments were repeated twice with similar results. TLR4, toll-like receptor 4; Min, minocycline. ${ }^{* * *} P<0.001,{ }^{*} P<0.05$, Significant difference with sham + vehicle group; \#\#\# $<0.001$, significant difference with CCI + Vehicle group. 
A comparison of WDR neurons in CCI and sham-operated rats showed significant increases in C-fibre $\left(t_{23}=-4.53, P<0.001\right)$; A $\delta$-fibre $\left(t_{23}=-3.69, P=0.001\right)$, post-discharge $\left(t_{23}=-3.76\right.$, $P=0.004)$, input spikes $\left(t_{23}=-2.2, P=0.047\right)$, and wind-up spikes $\left(t_{23}=-4.52 . P<0.001\right)$ (Fig. 3$)$. Moreover, when comparing $\mathrm{CCI}$ to the sham-operated group, a significant increase was observed in the WDR neurons response to the von Frey filament $60 \mathrm{~g}\left(t_{19}=-2.68, P=0.015\right)$ and noxious pinch stimulus $\left(t_{19}=-3.39, P=0.001\right)$. There was also a significant increase in the evoked responses of WDR neurons to noxious thermal stimuli of $45^{\circ} \mathrm{C} \quad\left(\mathrm{t}^{13}=-2.85, \quad P=0.014\right)$ and $50^{\circ} \mathrm{C}$ $\left(t_{13}=-7.77, P<0.001\right)$ (Fig. 4)

Effect of post-injury treatment with minocycline on the electrical and natural evoked responses of WDR neurons

The effect of post-injury administration of minocycline $(40 \mathrm{mg} /$ $\mathrm{kg}$ ) was evaluated on POD 14, and a total of 12 WDR neurons were employed. The results showed significant decreases in $\mathrm{C}$ fibre $\left(t_{17}=2.12, \quad P=0.049\right) \quad$ and $\mathrm{A} \delta$-fibre $\left(t_{17}=2.51\right.$, $P=0.022)$ mediated transmission to WDR neurons, as well as

(a)

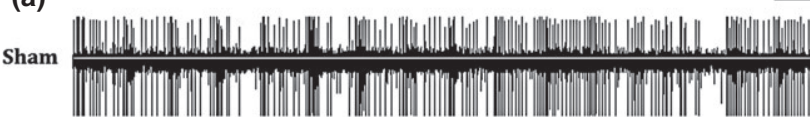
CCI

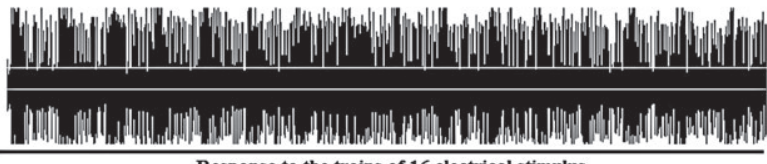

(b)

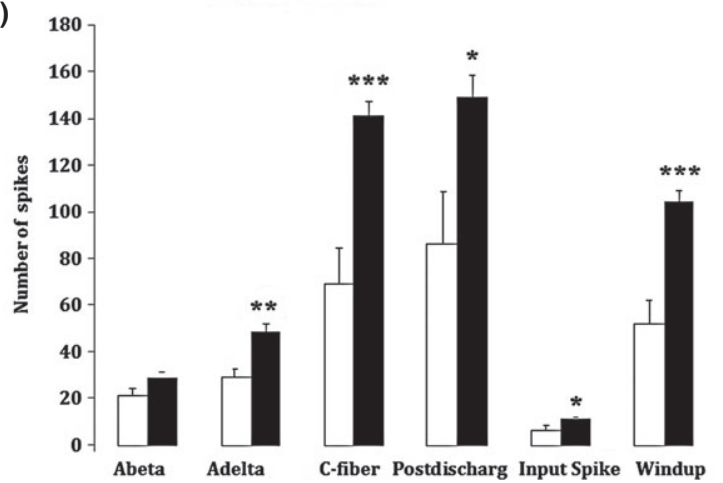

Fig. 3 The responses of WDR neurons to electrical, mechanical and thermal stimuli in CCI and sham operated groups. (a) Two traces of WDR neuron responses to a train of 16 electrical stimuli $(0.5 \mathrm{~Hz}$, duration $2 \mathrm{~ms}$ at amplitude 3-times the $\mathrm{C}$-fibre threshold) in sham and $\mathrm{CCI}$ rats were presented. The responses evoked by the different fibres were quantified on the basis of latency measurements (A $\beta$-fibre, $\mathrm{A} \delta$-fibre, and $\mathrm{C}$-fibre). Any neuronal responses occurring after the $\mathrm{C}$-fibre latency band were taken to be the post-discharge of the neuron (PD). Input spike (IS) is the neuronal responses occurring in the C-fibre and PD Latency band in first stimulus. (b) Wind-up is calculated by, subtracting the (Input Spikes $\times 16$ ) from the final cumulative $\mathrm{C}$ and PD spikes. Results are presented as mean $\pm \operatorname{SEM}(n=9-14$ unit/group). The A $\delta$ and C-fibre transmission onto WDR neurons were increased in CCI rats compared to sham group, as well as PD, IS and Wind-up of WDR neurons. ${ }^{*} P<0$. $05, * * P<0.01, * * * P<0.001$, significant difference with sham group. $(\square)$, Sham; (ם), CCI post-discharge $\left(t_{17}=2.42, \quad P=0.023\right)$ and wind-up spikes $\left(t_{17}=3.95, P=0.001\right)$ in comparison to the CCI group (Fig. 5).

In addition, the mechanically evoked responses of WDR neurons $(n=10)$, to von Frey filament $60 \mathrm{~g}\left(t_{15}=4.16, P<0.001\right)$ and noxious pinch $\left(t_{15}=4.15 . P<0.001\right)$ showed considerable decreases in comparison to CCI rats. However, there was no noticeable differences in responses to von Frey filaments 2 and 8 g (Fig. 6a).

In addition, the administration of minocycline significantly decreased the evoked responses of WDR neurons to noxious thermal stimuli at $45^{\circ} \mathrm{C}\left(t_{15}=3.51, \quad P<0.001\right)$ and $50^{\circ} \mathrm{C}$ $\left(t_{15}=9.61, P<0.001\right)$ when compared to CCI rats. But there was no significant difference in the case of innocuous thermal stimuli, or at 35 or $40^{\circ} \mathrm{C}$ (Fig. 6b).

\section{DISCUSSION}

The main causes of painful neuropathy are still unknown; many studies have established that microglia are involved in the initiation and maintenance of chronic and neuropathic pain. Additionally, inhibition of microglial activity effectively attenuates the behavioral symptoms of neuropathic pain. ${ }^{8,26,27}$

The findings of this study showed that after CCI with increasing thermal hyperalgesia, the expression of TLR4 and hypersensitivity of WDR neurons in spinal cord significantly increased. However, it is important to note that sham operated rats did not show significant changes in hyperalgesia and TLR4 expression, as well as WDR neuron hypersensitivity. This suggests that the changes observed in the CCI rats are not due to the surgical exposure alone. Tanga et al. (2004) and Hutchinson et al. (2008) show increased TLR4 mRNA and protein expression of lumbar spinal cord with enhanced pain in spinal nerve ligation and CCI models of neuropathic pain. ${ }^{16,28}$ In the nervous system, TLR4 is almost exclusively expressed by microglial cells. ${ }^{29,30}$ Owens et al $^{14}$ reported that in response to nerve injury, TLR1, TLR2, and TLR4 were up-regulated in the CNS and led to the production of pro-inflammatory cytokines (TNF, IL-1) and chemokines. Pro-inflammatory cytokines have been implicated in central sensitization, hence the development and maintenance of persistent pain states. $3,31,32$

In addition, the results of this study showed that post-injury administration of minocycline could effectively attenuate thermal hyperalgesia and decrease TLR4 expression. Raghavendra et al. (2003) showed that minocycline was a potent inhibitor of microglial activation, was effective in reducing nerve injury-induced mechanical allodynia, and decreased the mRNA expression of ITGAM and TLR4. However, when minocycline was administered 5 days after nerve injury, the behavioral hypersensitivity was unaffected but the mRNA expression for ITGAM and TLR4 was decreased. ${ }^{26}$ Taken together, this suggests that microglia are the initial immunoeffector cell sensor that, if inhibited prior to the onset of astrocytic activation, may decrease peripheral injuryinduced hypersensitivity. ${ }^{6,16}$ Otherwise, Mei et al. (2011) reported that post-injury intrathecal administration of a single dose of minocycline on days 1,3 , and 7 after spinal nerve ligation-induced neuropathic pain in rat effectively alleviates mechanical allodynia together with inhibition of microglial activity. ${ }^{33}$ However, our results showed that repeated administration of minocycline, from days 6 to 14 after nerve injury, could effectively 
(a)
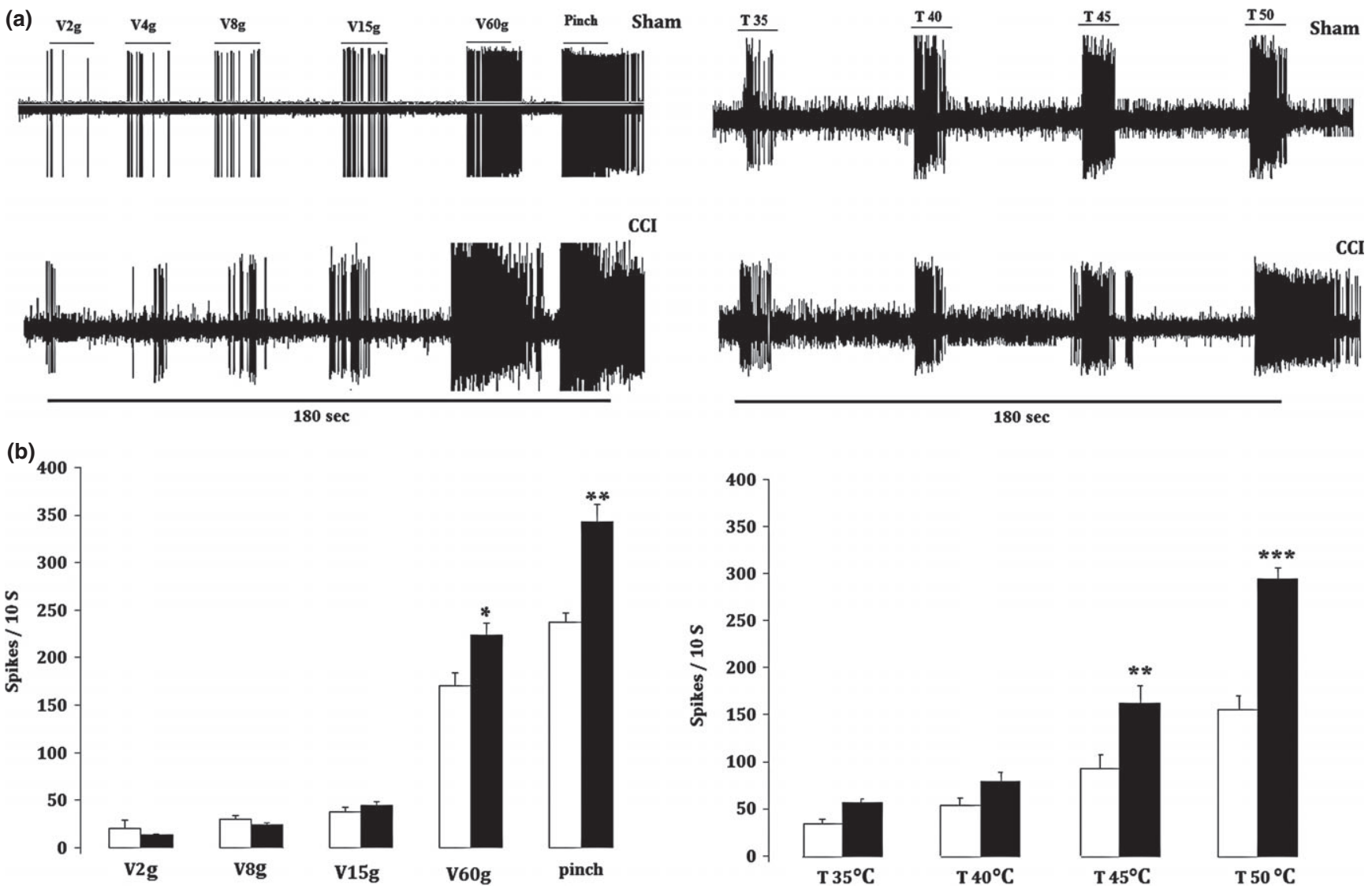

Fig. 4 The response of WDR neurons to natural (mechanical and thermal) stimulus in CCI and sham operated groups $(n=8)$. (a) Traces of WDR neurons response to mechanical stimulus (V2 g, V8 g, V15 g, V60 g and pinch) and thermal stimulus (35, 40, 45 and 50 $\left.{ }^{\circ} \mathrm{C}\right)$ were represented. Each stimulus applied for $10 \mathrm{~s}$ at the most sensitive point of the WDR neuron receptive field. (b) Post-stimulus time histograms (PSTH) for this stimulus were calculated. Results show as mean $\pm \operatorname{SEM}(n=9-12$ unit/group). The responses of WDR neurons to noxious thermal and mechanical stimulus were increased in CCI groups compared to the sham group. ${ }^{*} P<0.05,{ }^{* *} P<0.01,{ }^{* * *} P<0.001$, significant difference with sham group; WDR, wide dynamic range neuron; g, gram.

attenuate thermal hyperalgesia and reduce microglial activity state as shown by decreases in TLR4 expression.

In the electrophysiological part of this study, the frequencies of WDR neuron responses were recorded. The receptive fields of all neurons defined in the toe/plantar region of the ipsilateral hind paw and the responses to both noxious and innocuous stimuli. The results showed that the responses of WDR neurons, to $\mathrm{A} \delta$ - and C-fibre spikes, post-discharge (PD) and wind up spikes, remarkably increased in CCI animals compared to the sham group. In addition, the evoked responses of WDR neurons to noxious, thermal, and mechanical stimuli were significantly increased in CCI rats.

Pitcher $e t a l .{ }^{34}$ reported that the responses of WDR neurons to noxious, mechanical, and thermal stimuli were increased after ligation of the sciatic nerve. Le Blanch et al. ${ }^{35}$ showed that after $\mathrm{CCI}$, the spontaneous activity and evoked responses of WDR neurons to mechanical and thermal noxious stimuli increased significantly. Woolf et $a l^{36}$ reported that the responses of WDR neurons to noxious, mechanical, and thermal stimuli considerably increased in CCI, and this can be associated with decreases in GABA activity and disinhibition in the spinal cord, increasing NMDA activity and synaptic plasticity, ${ }^{37}$ or expression of abnormal sodium channels in pre- and post-synaptic neurons. ${ }^{38}$ The evidence explains the neuronal basis for the behavioral signs of allodynia and hyperalgesia in neuropathy. ${ }^{25}$ However, increases in the production of pro-inflammatory cytokines from activated microglial cells, as evidenced here by increases in TLR4 expression, may contribute to the spontaneous activity in intact nerve fibres. Specifically, changes in C-fibre activity are responsible for behavioral and electrophysiological changes in neuropathic pain. ${ }^{2,39-41}$ On the other hand, post-injury administration of minocycline $(40 \mathrm{mg} / \mathrm{kg}$; i.p.) significantly decreased the frequency of the WDR neuron response to electrical, mechanical, and thermal stimuli. This is in agreement with many previous behavioral studies that collectively verify the effectiveness of minocycline for the treatment of neuropathic pain. ${ }^{611,27,42}$ Owolabi and Saab reported that in SNL-induced neuropathic pain, the response of WDR neurons to noxious pinch increased, and the administration of a single dose of minocycline significantly reduced the response of WDR neurons to pinch. ${ }^{43}$ In addition, the results of this study demonstrated that the responses of the WDR neurons to innocuous, mechanical, and thermal stimulus displayed minor differences between the sham, CCI, and minocycline groups. Consistent with these results, other electrophysiological studies also demonstrate that the responses of WDR neurons to innocuous stimuli do not change after nerve injury. ${ }^{19,44}$ These findings 

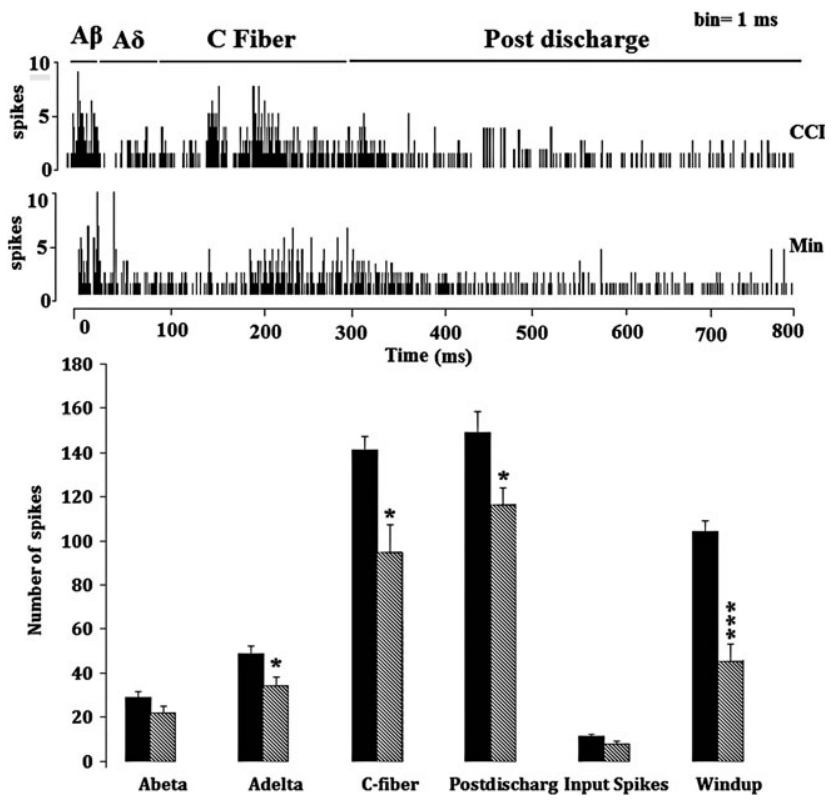

Fig. 5 Minocycline inhibited hyper-responsiveness of WDR neurons in CCI rats. Minocycline $40 \mathrm{mg} / \mathrm{kg}$ inhibited A $\delta$ and C-fiber mediated transmission onto WDR neurons $(n=10)$ compared to CCI rats $(n=12)$. Minocycline also had a significant inhibitory effect on WDR neuronal post-discharge (PD). Minocycline inhibited wind-up, a potentiated response mediated by nociceptive C-fiber activity and a measure of neuronal hyperexcitability. The inhibitory effects of minocycline were not extended to $\mathrm{A} \beta$-evoked responses since there was no difference in $\mathrm{A} \beta$ evoked responses between sham, CCI or minocycline rats. Post-stimulus time histogram (PSTH) for CCI and minocycline were represented on top. ${ }^{*} P<0.05,{ }^{*} * P<0.001$, significant difference with CCI group. (ם), $\mathrm{CCI} ;(\mathbb{\mathbb { N }}), \mathrm{Min}$ suggest that nerve injury mostly affects synaptic transmission in the neurons of pain pathways.

In conclusion, the results of this study indicate that post-injury repeated administration of minocycline is an effective way for treating neuropathic pain, and the underlying mechanisms are suppressing spinal microglia activation and reducing WDR neuron hyper-responsiveness. This study provides a new and exact strategy for treating neuropathic pain by post-injury modulation of microglial activity.

\section{MATERIALS AND METHODS}

\section{Animals}

Seventy male Wistar rats (Pasture Institute, Tehran, Iran) weighing $230 \pm 30 \mathrm{~g}$ (at the time of surgery) were used in this study. All animals were housed under standardized conditions in a room on a $12 \mathrm{~h}$ light/dark cycle with food and water available ad libitum. All experimental procedures followed the Guidelines on Ethical Standards for Investigations of Experimental Pain in Animals, ${ }^{45}$ and were also approved by the Research and Ethics Committee of Shahid Beheshti University of Medical Sciences.

\section{Protocol}

Animals were randomly divided into the following eight groups: (i) a sham-operated group; (ii) a group subjected to CCI and injected with normal saline (CCI group); and (iii) three groups subjected to CCI and injected with minocycline $(10,20,40 \mathrm{mg} /$ $\mathrm{kg}$; i.p.). There were eight rats in each group. The behavioral test was performed in the morning, 1 day before surgery and 2, 6, 10, and 14 days after surgery by the same person. Finally,
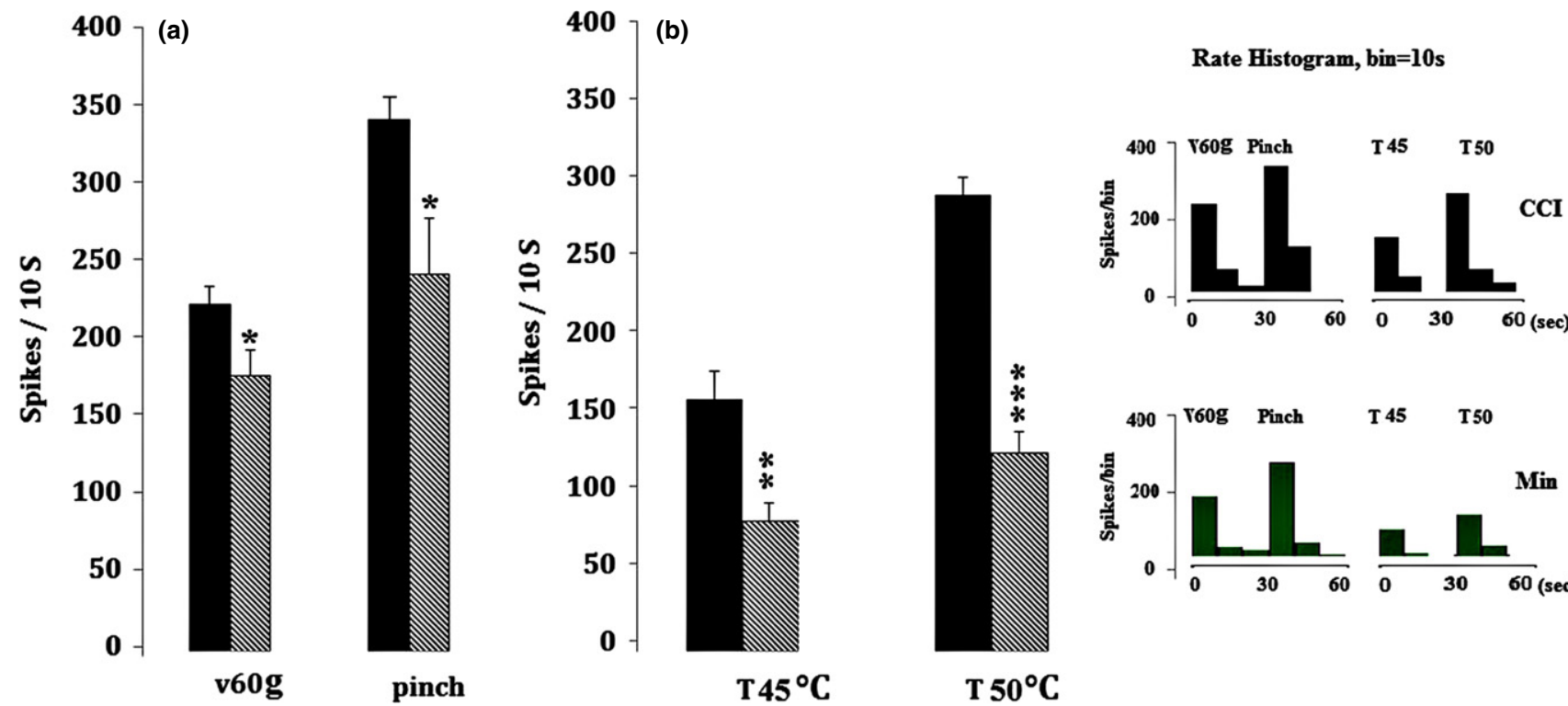

Fig. 6 Minocycline inhibits the responses of WDR neurons to noxious mechanical and thermal stimuli in CCI rats. Minocycline $(n=10)$ was effective in inhibiting neuronal responses to (a) noxious mechanical $\left(60 \mathrm{~g}\right.$ and pinch); and (b) thermal $\left(45,50^{\circ} \mathrm{C}\right.$ stimuli when compared to CCI rats $(n=12)$. The inhibitory effects of Minocycline were not extended to innocuous mechanical $(8,15,26 \mathrm{~g})$ and thermal $\left(35,40^{\circ} \mathrm{C}\right)$ evoked responses $($ data not shown), as there were no differences in these responses between sham, CCI and minocycline groups. ${ }^{*} P<0.05, * * P<0.01,{ }^{* * *} P<0.001$, significant difference

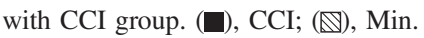


lumbar segments of the spinal cord were collected for Western blot analysis of TLR4 expression. In the electrophysiological part, 30 rats were randomly divided into the following groups: (i) a sham-operated group; (ii) a group subjected to CCI and injected with normal saline (CCI group); (iii) a group subjected to CCI and injected with minocycline (40 mg/kg; i.p.).

\section{Surgical operation}

Chronic constriction injury (CCI) of the sciatic nerve was performed according to the method described by Bennett and Xie. ${ }^{46}$ Briefly, rats were anesthetized by an i.p. injection of sodium pentobarbital $(60 \mathrm{mg} / \mathrm{kg})$, and the sciatic nerve was exposed at midthigh by dissecting through the biceps femoris muscle proximal to the sciatic trifurcation, and four loose ligatures using chromic gut 4-0 thread were tied around the nerve at $1 \mathrm{~mm}$ intervals until a fine twitch was observed in the respective hind limb as previously described. ${ }^{46}$ The muscle and skin were closed in layers using 4-0 silk thread. In sham-operated controls, an identical surgical procedure was performed, except the sciatic nerve was not ligated.

\section{Drug administration}

Minocycline hydrochloride (Sigma, St Louis, MO, USA) was dissolved in normal saline and administered i.p. at doses of 10, 20 , and $40 \mathrm{mg} / \mathrm{kg}$ in the CCI groups, from postoperative day (POD) 6 to POD 14, once daily; sham and CCI groups received saline as a vehicle (eight rats/group). Sixty minutes after administration of the last dose of minocycline or saline on POD 14, behavioral, molecular, and electrophysiological studies were performed.

\section{Behavioral study}

Thermal hyperalgesia was assessed by means of a Hargreaves test with paw withdrawal latency (PWL) as previously described. ${ }^{27}$ Briefly, the rats were placed in a clear plastic container on an elevated floor of clear, heat-tempered glass (Plantar Test, Ugo Basile, Monvalle, Italy). After a $15 \mathrm{~min}$ period of habituation, a radiant heat source $(50 \mathrm{~W}$ halogen reflector bulbs with intensity controlled by a constant voltage source) was focused on the plantar surface of the ipsilateral and contralateral hind paw. Each paw was tested five times at 5 min intervals, and the average value of the withdrawal latency of five consecutive tests was recorded. The cut-off time in the absence of a response was $33 \mathrm{~s}$ to avoid tissue damage.

\section{Western blotting}

After the last dose of minocycline or vehicle on POD 14, the animals were killed under isoflurane anesthesia, and the lumbar (L5-L6) regions of the spinal cords were rapidly removed. Tissue samples were homogenized (Brinkmann Polytron Homogenizer; $20000 \mathrm{rpm}$ for $30 \mathrm{~s}$ at $4^{\circ} \mathrm{C}$; Kinematica $\mathrm{AG}$, Lucerne, Switzerland $)$ in RIPA buffer $((50 \mathrm{mmol} / \mathrm{L}$ Tris-HCl, $\mathrm{pH} 7.5$, $150 \mathrm{mmol} / \mathrm{L} \mathrm{NaCl}, 1 \mathrm{mmol} / \mathrm{L}$ ethylenediamine tetra acetic acid (EDTA), $1 \%$ NP40, $0.5 \%$ sodium dodecyl sulfate (SDS), $1 \mathrm{mmol} / \mathrm{L}$ sodium orthovanadate, $2.5 \mu \mathrm{g} / \mathrm{mL}$ aprotinin, $2 \mu \mathrm{g} / \mathrm{mL}$ leupetin, $2 \mu \mathrm{g} / \mathrm{mL}$ pepstatin A), and cleared by centrifugation $\left(10000 \times g, 15^{\circ} \mathrm{C}\right.$ for $\left.10 \mathrm{~min}\right)$. The protein concentration in the supernatant was determined using a Bradford assay. ${ }^{47}$ Samples containing $60 \mu \mathrm{g}$ of protein were heated for $8 \mathrm{~min}$ at $99^{\circ} \mathrm{C}$ in loading buffer (4\% SDS, $25 \mathrm{mmol} / \mathrm{L}$ Tris- $\mathrm{HCl}, \mathrm{pH}$ 6.8, 5\% glycerol, $0.5 \%$ 2-mercaptoethanol, $0.01 \%$ bromophenol blue) and resolved by SDS-PAGE on $10 \%$ polyacrylamide gels $(120 \mathrm{~V}$ for $60 \mathrm{~min}$ ). After gel electrophoresis, the proteins were electrophoretically transferred to PVDF membranes (Millipore; Bedford, MA, USA) using the Mini-PROTEAN II (Bio-Rad, Hercules, CA, USA) at $100 \mathrm{~V}$ for $85 \mathrm{~min}$. Nonspecific binding sites were blocked using $2 \%$ blocking buffer $(0.2 \%$ Aurora blocking reagent) in TBST (Tris-buffered saline with $0.1 \%$ Tween-20) for $90 \mathrm{~min}$ at $24^{\circ} \mathrm{C}$. The blots were incubated overnight at $4^{\circ} \mathrm{C}$ with primary antibodies in the blocking buffer (mouse monoclonal anti-TLR4, 1:2000 dilution; Abcam, Cambridge, UK). After three 10-min washes in TBS and $0.1 \%$ Tween-20, blots incubated with the appropriate peroxidase-conjugated secondary antibody in blocking buffer, anti-rabbit $\operatorname{IgG}(1: 10000$, Abcam) for $1 \mathrm{~h}$ at room temperature. Then, following three 10 -min washes in TBS and $0.1 \%$ Tween-20, and one wash in TBS, the immunoreactivity of the proteins located on the membrane was visualized using a chemiluminescence detection system (ECL advance; Amersham, Freiburg, Germany). The membranes were stripped in stripping buffer $(100 \mu \mathrm{mol} / \mathrm{L}$ 2-mercaptoethanol, $2 \%$ SDS, $62.5 \mathrm{mmol} / \mathrm{L}$ Tris; $\mathrm{pH}$ 6.7) at $50^{\circ} \mathrm{C}$ for $30 \mathrm{~min}$ and re-probed with primary antibody against $\beta$-actin (1:5000; cell signaling) as a loading control. The densities of target protein blots and $\beta$-actin immunoreactive bands were analyzed using Image J software (V1.41; NIH, Bethesda, MD, USA) after background subtraction. Target protein levels were normalized against $\beta$-actin levels and expressed as relative fold changes compared to the sham group.

\section{Electrophysiological recording}

In vivo electrophysiological recording was performed at day 14 after induction of CCI neuropathic pain. Animals were anesthetized with $2.0-2.5 \%$ isoflurane in $66 \% \mathrm{~N}_{2} \mathrm{O}$ and $33 \% \mathrm{O}_{2}$ gaseous mixture until areflexia was produced. Rats were secured in a stereotaxic frame and a laminectomy was performed on the lumbar vertebrae $\mathrm{L}_{1}-\mathrm{L}_{3}$ to expose segments $\mathrm{L}_{4}-\mathrm{L}_{5}$ of the spinal cord. The dura was removed and the spine was held firmly in place by clamps, caudal and rostral to the laminectomy. The isoflurane concentration was lowered (1.5-2\%) while surgery was performed, and then held at $1-1.2 \%$ for the duration of the experiment. This level of anesthesia maintained areflexia. The rats breathed spontaneously at an average rate of 110 breaths per minute and their circulatory system was not compromised. Throughout the experiment, the core body temperature of the rat was monitored and maintained $\left(36.5-37.5^{\circ} \mathrm{C}\right)$ by means of a heating blanket connected to a rectal thermal probe via an automatic feedback control unit (Borje Sanat Co. Tehran, Iran). At the end of the experiment, the rats were killed with an overdose of isoflurane.

A parylene-coated tungsten microelectrode was lowered directly into the cord using a SCAT Microdrive (Digitimer, Welwyn Garden City, UK) that enabled researchers to estimate the depth of the neuron from the dorsal surface of the spinal cord. In $\mathrm{CCI}$ and sham-operated animals, neurons ipsilateral to the ligated 
hind paw were recorded. Neurons used in this experiment were located at depths of $300-1000 \mu \mathrm{m}$ from the dorsal surface of the spinal cord, with defined receptive fields in the toe regions and plantar surface of the hind paw. Spontaneously active neurons with no clear receptive fields were not included in the study. Extracellular single unit recordings were made from WDR neurons of the dorsal horn that responded to both innocuous (brush) and noxious (pinch) stimuli applied to the receptive fields in the plantar region of the hind paw. All neurons included in this study were responsive to electrical stimulation of the A- and C-fibres based on a post-stimulus time histogram that was constructed as previously described. ${ }^{48}$

The peripheral neuronal receptive field was stimulated using a range of noxious and innocuous natural stimuli, mechanical punctuate (von Frey filament 2-60 g, and pinch) and heat (35$50^{\circ} \mathrm{C}$ ), over a period of $10 \mathrm{~s}$. Heat was applied with a constant water jet onto the centre of the receptive field. A time interval of $1 \mathrm{~min}$ was left between stimulation to prevent sensitization. The spontaneous activity of the neuron was taken into account by recording the number of action potentials over a $15 \mathrm{~s}$ period prior to the stimulation by the mechanical and thermal stimuli. This 'background measurement' was then subtracted from the response to each of the stimuli. In addition, the number of action potentials evoked by the $35^{\circ} \mathrm{C}$ water was subtracted from the number of action potentials evoked by the 40, 45, and $50^{\circ} \mathrm{C}$ water to determine the response of the WDR neurons to heat.

Electrical stimulation consisted of a train of 16 transcutaneous electrical stimuli $(2 \mathrm{~ms}$ wide pulses, $0.5 \mathrm{~Hz})$ that were applied by means of two pins inserted into the center of the receptive field, at three times the threshold current for the C-fibre responses. A histogram of post-stimulus time was constructed and the responses of different fibres were separated according to their latencies (A $\beta$-fibre $0-20 \mathrm{~ms}$; A $\delta$-fibre $20-90 \mathrm{~ms}$; and $\mathrm{C}$ fibre 90-300 ms). Responses occurred after the C-fibre latency was characterized as post-discharge (300-800 ms). Wind-up was calculated as the total number of action potentials evoked after 16 stimuli at three times the $\mathrm{C}$-fibre threshold minus the input spike multiplied by 16 . Input spike was the number of $\mathrm{C}$-fibre latencies elicited by the first electrical stimulus. All activity was amplified and filtered at $0.3-5 \mathrm{kHz}$ (Electromodule; Science Beam, Tehran, Iran), and fed directly into the data acquisition unit (Electromodule; Science Beam) and stored on a Pentium 4 computer in order to construct the wave forms or plot the poststimulus time histograms. The data were analyzed off-line using Plexon Off-Line Sorter (version 2.88; Plexon Inc, Dallas, TX, USA) and NeuroExplorer (version 4.109, Nex Inc, Madison, AL, USA).

\section{Statistics analysis}

Data were expressed as the mean \pm SEM. GraphPad Prism (version 5.0; GraphPad Prism Software Inc., San Diego, CA, USA) was used for statistical analysis. Data from the Western blot analysis were compared by one-way ANOvA, followed by a Tukey's post-hoc test. Independent samples T-test was used to compare the electrophysiological data between sham and CCI or CCI and minocycline group. $P$-values less than 0.05 were considered as statistically significant.

\section{ACKNOWLEDGMENTS}

This work was financially supported by the Iran National Science Foundation (INSF) and Neuroscience Research Center of Shahid Beheshti University of Medical Sciences. We are grateful to acknowledge Mr. Nematollah Shomoossi for their editorial assistance.

\section{REFERENCES}

1. Zimmermann M. Pathobiology of neuropathic pain. Eur. J. Pharmacol. 2001; 429: 23-37.

2. Clark AK, Old EA, Malcangio M. Neuropathic pain and cytokines: Current perspectives. J. Pain Res. 2013; 6: 803-14.

3. Watkins LR, Milligan ED, Maier SF. Glial activation: A driving force for pathological pain. Trends Neurosci. 2001; 24: 450-5.

4. Bradesi S. Role of spinal cord glia in the central processing of peripheral pain perception. Neurogastroenterol. Motil. 2010; 22: 499-511.

5. Tsuda M, Beggs S, Salter MW, Inoue K. Microglia and intractable chronic pain. Glia 2013; 61: 55-61.

6. Raghavendra V, Tanga F, DeLeo JA. Inhibition of microglial activation attenuates the development but not existing hypersensitivity in a rat model of neuropathy. J. Pharmacol. Exp. Ther. 2003; 306: 62430.

7. Hua XY, Svensson CI, Matsui T, Fitzsimmons B, Yaksh TL, Webb M. Intrathecal minocycline attenuates peripheral inflammationinduced hyperalgesia by inhibiting p38 MAPK in spinal microglia. Eur. J. Neurosci. 2005; 22: 2431-40.

8. Ledeboer A, Sloane EM, Milligan ED, et al. Minocycline attenuates mechanical allodynia and proinflammatory cytokine expression in rat models of pain facilitation. Pain 2005; 115: 71-83.

9. Wu J, Xu Y, Pu S, Jiang W, Du D. p38/MAPK inhibitor modulates the expression of dorsal horn $\mathrm{GABA}(\mathrm{B})$ receptors in the spinal nerve ligation model of neuropathic pain. NeuroImmunoModulation 2011; 18: $150-5$.

10. Mika J. Modulation of microglia can attenuate neuropathic pain symptoms and enhance morphine effectiveness. Pharmacol. Rep. 2008; 60: 297-307.

11. Mika J, Osikowicz M, Rojewska E et al. Differential activation of spinal microglial and astroglial cells in a mouse model of peripheral neuropathic pain. Eur. J. Pharmacol. 2009; 623: 65-72.

12. Hashizume H, DeLeo JA, Colburn RW, Weinstein JN. Spinal glial activation and cytokine expression after lumbar root injury in the rat. Spine 2000; 25: 1206-17.

13. Bettoni I, Comelli F, Rossini C et al. Glial TLR4 receptor as new target to treat neuropathic pain: Efficacy of a new receptor antagonist in a model of peripheral nerve injury in mice. Glia 2008; 56: 1312-19.

14. Owens T, Babcock AA, Millward JM, Toft-Hansen H. Cytokine and chemokine inter-regulation in the inflamed or injured CNS. Brain Res. Rev. 2005; 48: 178-84.

15. Tanga FY, Nutile-McMenemy N, DeLeo JA. The CNS role of Tolllike receptor 4 in innate neuroimmunity and painful neuropathy. Proc. Natl Acad. Sci. USA 2005; 102: 5856-61.

16. Tanga FY, Raghavendra V, DeLeo JA. Quantitative real-time RTPCR assessment of spinal microglial and astrocytic activation markers in a rat model of neuropathic pain. Neurochem. Int. 2004; 45: 397-407.

17. Suzuki R, Hunt SP, Dickenson AH. The coding of noxious mechanical and thermal stimuli of deep dorsal horn neurones is attenuated in NK1 knockout mice. Neuropharmacology 2003; 45: 1093-100.

18. Suzuki R, Kontinen VK, Matthews E, Williams E, Dickenson AH. Enlargement of the receptive field size to low intensity mechanical stimulation in the rat spinal nerve ligation model of neuropathy. Exp. Neurol. 2000; 163: 408-13. 
19. Suzuki R, Chapman V, Dickenson AH. The effectiveness of spinal and systemic morphine on rat dorsal horn neuronal responses in the spinal nerve ligation model of neuropathic pain. Pain 1999; 80: 215-28.

20. Gwak YS, Hulsebosch CE. Remote astrocytic and microglial activation modulates neuronal hyperexcitability and below-level neuropathic pain after spinal injury in rat. Neuroscience 2009; 161: 895903.

21. Bahari Z, Manaheji H, Hosseinmardi $\mathrm{N}$ et al. Induction of spinal long-term synaptic potentiation is sensitive to inhibition of neuronal nos in 15 spinal nerve-transected rats. EXCLI J. 2014; 13: 751-60.

22. Behbehani MM, Dollberg-Stolik O. Partial sciatic nerve ligation results in an enlargement of the receptive field and enhancement of the response of dorsal horn neurons to noxious stimulation by an adenosine agonist. Pain 1994; 58: 421-8.

23. Takaishi K, Eisele JH Jr, Carstens E. Behavioral and electrophysiological assessment of hyperalgesia and changes in dorsal horn responses following partial sciatic nerve ligation in rats. Pain 1996; 66: 297-306.

24. Pertovaara A, Kontinen VK, Kalso EA. Chronic spinal nerve ligation induces changes in response characteristics of nociceptive spinal dorsal horn neurons and in their descending regulation originating in the periaqueductal gray in the rat. Exp. Neurol. 1997; 147: 428-36.

25. Yakhnitsa V, Linderoth B, Meyerson BA. Spinal cord stimulation attenuates dorsal horn neuronal hyperexcitability in a rat model of mononeuropathy. Pain 1999; 79: 223-33.

26. Raghavendra V, Tanga F, Rutkowski MD, DeLeo JA. Anti-hyperalgesic and morphine-sparing actions of propentofylline following peripheral nerve injury in rats: Mechanistic implications of spinal glia and proinflammatory cytokines. Pain 2003; 104: 655-64.

27. Nazemi S, Manaheji H, Zaringhalam J, Sadeghi M, Haghparast A. Post-injury repeated administrations of minocycline improve the antinociceptive effect of morphine in chronic constriction injury model of neuropathic pain in rat. Pharmacol. Biochem. Behav. 2012; 102: 520-5.

28. Hutchinson MR, Zhang $\mathrm{Y}$, Brown $\mathrm{K}$ et al. Non-stereoselective reversal of neuropathic pain by naloxone and naltrexone: Involvement of toll-like receptor 4 (TLR4). Eur. J. Neurosci. 2008; 28: 20 9.

29. Laflamme N, Rivest S. Toll-like receptor 4: The missing link of the cerebral innate immune response triggered by circulating gram-negative bacterial cell wall components. FASEB J. 2001; 15: 155-63.

30. Lehnardt S, Lachance $\mathrm{C}$, Patrizi $\mathrm{S}$ et al. The toll-like receptor TLR4 is necessary for lipopolysaccharide-induced oligodendrocyte injury in the CNS. J. Neurosci. 2002; 22: 2478-86.

31. Sorkin LS, Doom CM. Epineurial application of TNF elicits an acute mechanical hyperalgesia in the awake rat. J. Peripher. Nerv. Syst. 2000; 5: 96-100.

32. Watkins LR, Milligan ED, Maier SF. Spinal cord glia: New players in pain. Pain 2001; 93: 201-5.
33. Mei XP, Xu H, Xie $\mathrm{C}$ et al. Post-injury administration of minocycline: An effective treatment for nerve-injury induced neuropathic pain. Neurosci. Res. 2011; 70: 305-12.

34. Pitcher GM, Henry JL. Cellular mechanisms of hyperalgesia and spontaneous pain in a spinalized rat model of peripheral neuropathy: Changes in myelinated afferent inputs implicated. Eur. J. Neurosci. 2000; 12: 2006-20.

35. LeBlanc BW, Zerah ML, Kadasi LM, Chai N, Iwata M, Saab CY. Pain-induced brain plasticity is reversed by pharmacology and neurotechnology. In: New York Academy of Sciences (ed.). Conference Proceedings of Chronic Inflammatory and Neuropathic Pain, 2-3 June 2011, New York, NY, USA. 2011; 2; 925.

36. Woolf CJ, Salter MW. Neuronal plasticity: Increasing the gain in pain. Science 2000; 288: 1765-9.

37. Dickenson AH, Chapman V, Green GM. The pharmacology of excitatory and inhibitory amino acid-mediated events in the transmission and modulation of pain in the spinal cord. Gen. Pharmacol. 1997; 28: 633-8.

38. Waxman SG, Dib-Hajj S, Cummins TR, Black JA. Sodium channels and pain. Proc. Natl Acad. Sci. USA 1999; 96: 7635-9.

39. Clark AK, Gentry C, Bradbury EJ, McMahon SB, Malcangio M. Role of spinal microglia in rat models of peripheral nerve injury and inflammation. Eur. J. Pain 2007; 11: 223-30.

40. Gautron M, Jazat F, Ratinahirana H, Hauw JJ, Guilbaud G. Alterations in myelinated fibres in the sciatic nerve of rats after constriction: Possible relationships between the presence of abnormal small myelinated fibres and pain-related behaviour. Neurosci. Lett. 1990; 111: $28-33$.

41. Liu F, Yuan H. Role of glia in neuropathic pain. Front Biosci. 2014; 19: 798-807.

42. Mika J, Osikowicz M, Makuch W, Przewlocka B. Minocycline and pentoxifylline attenuate allodynia and hyperalgesia and potentiate the effects of morphine in rat and mouse models of neuropathic pain. Eur. J. Pharmacol. 2007; 560: 142-9.

43. Owolabi SA, Saab CY. Fractalkine and minocycline alter neuronal activity in the spinal cord dorsal horn. FEBS Lett. 2006; 580: 430610 .

44. Suzuki R, Rahman W, Hunt SP, Dickenson AH. Descending facilitatory control of mechanically evoked responses is enhanced in deep dorsal horn neurones following peripheral nerve injury. Brain Res. 2004; 1019: 68-76.

45. Zimmermann M. Ethical guidelines for investigations of experimental pain in conscious animals. Pain 1983; 16: 109-10.

46. Bennett GJ, Xie YK. A peripheral mononeuropathy in rat that produces disorders of pain sensation like those seen in man. Pain 1988; 33: $87-107$.

47. Bradford MM. A rapid and sensitive method for the quantitation of microgram quantities of protein utilizing the principle of protein-dye binding. Anal. Biochem. 1976; 72: 248-54.

48. Flatters SJ, Fox AJ, Dickenson AH. Nerve injury induces plasticity that results in spinal inhibitory effects of galanin. Pain 2002; 98: 249-58. 